\title{
An Analytical Survey on Routing Protocols for Wireless Sensor Network (WSN)
}

\author{
Khawar lqbal Malik \\ The University of Lahore \\ Sargodha, Campus. Sargodha
}

\begin{abstract}
Routing protocols in wireless sensor network is used for selection of best route to fulfill the different criteria like shortest route selection, congestion control, efficient data delivery, minimizing traffic delay and reliability. This paper presents the architecture of wireless sensor network, their design issues and different routing protocols available for wireless sensor network. These protocols are classified in four different groups that are; data centric, hierarchal, location based and QoS based. Under the each category, their strength and limitations are discussed. Some open research issues of energy consumption, network stability period and its life time are also discussed.
\end{abstract}

\section{Keywords}

Wireless Sensor Network, Routing Protocols, Cluster head, Reliability, Network life time, Stability Period.

\section{INTRODUCTION}

Wireless Personal Area Network (WPANs) is used for transfer of information on small distance. Wireless sensor network uses wireless sensor nodes to observe the data and signals from surroundings and broadcast to wireless sink node. Theses sensor are tiny devices that consist of four basic units, sensing unit, processing unit, receiver and transmitter unit and power unit. Sensor nodes are very small in size and consume very low energy. These sensors used to monitor the environment. These sensors are normally in small size, low power, and low cost devices. Sensors also have capabilities of computing and communication as well. With advancement of microelectronics, theses sensors are becoming smaller in size with increasing computational power and communication range.

In variety of application, like combat field, under water, security, disaster management, inventory control, medical and health applications, vehicle tracking, nuclear, agriculture, flood detection, biochemical and industrial environment. Many sensor nodes deployed randomly or uniformly in required area. These nodes work collectively and monitor the environment. Sensor nodes collect data aggregate and transfer it to sink node via some specific route. In some applications sensor nodes are deployed on predetermined locations, for example in industry. While in other application they allow the random deployment. In such application nodes are thrown from air like in battle field. Normally these nodes are selforganizing. Data transmission is done by establishing the intermediate nodes from source (sensor node) to destination (sink node) with multiple links or hops. So WSN requires ad hoc network methods. But existing algorithms and protocols for ad hoc network are not fully suitable for wireless networks because of under explanations [1]:

- Ad hoc network and WSN are different in terms of numbers of nodes. Normally WSN have more and numerous order than ad hoc network.

\author{
M. Mateen Yaqoob \\ The University of Lahore \\ Sargodha, Campus. Sargodha
}

- In WSN, node deployment density in higher than ad hoc network.

- Failure rate of sensor node is higher in WSN.

- WSN having varied dynamic topology means it changes frequently because of higher node failure ratio.

- Ad hoc uses P2P model mostly while WSN uses broadcast methodology.

- In WSN sensor nodes have limited resources like power, memory and processing capabilities.

- In WSN we normally could not use the unique ID like IP address for sensor nodes, as these are more in number and unique ID for all node becomes overhead.

Continuously change in topology is important factor that continuously affect the routing protocols. Sensor nodes normally change their data rout because of nodes mobility, node died, pre and post deployment of nodes in network, change in environment.

\section{WIRELESS SESOR NETWORK ARCHITECTURE}

A sensor network architecture design is influenced by many factors, which include:

Scalability: Scalability measures the density of the sensor nodes

Fault tolerance: is the ability to sustain sensor network functionalities without any interruption due to sensor node failures

Production costs: The cost of a single node is very important to justify the overall cost of the networks

Sensor network topology: Sensor network topology is very highly dynamic and change frequently.

Further thing that can prejudiced wireless sensor network architecture are, hardware constraints, power consumption, operating environment and transmission media [2].

Sensor nodes are normally spread in field area as shown in fig 1. Each node sense data and send out it back to sink. It can commune with task manager node through Internet or satellite [2] 


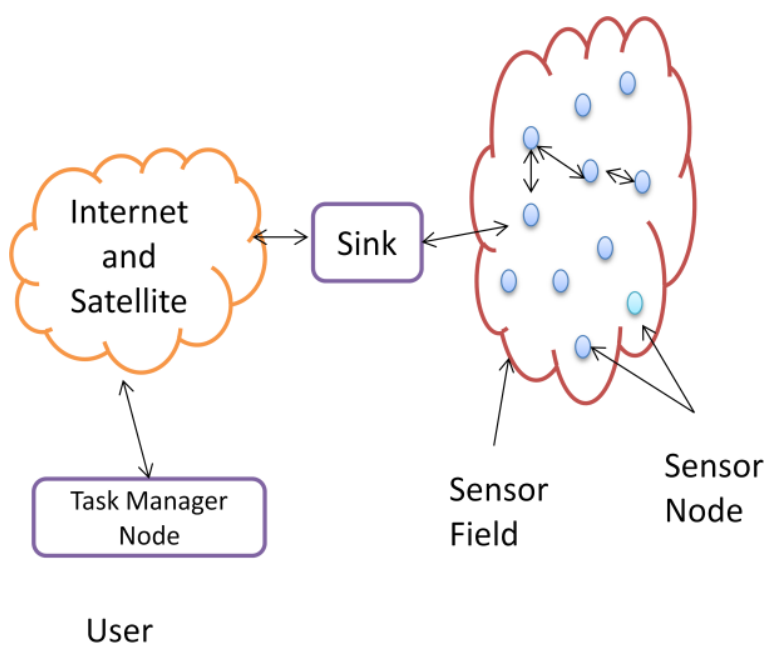

Fig 1: A typical sensor network with sin and task manager

\section{PROTOCOL STACK}

Protocol stack of WSN is shown in fig 2, this stack having of 5 layers from application on top and physical layer in bottom, just like TCP/IP stack and three planes for power management, mobility management and task management. On Application layer different kind of software apps can be developed as per sensing requirement. Transport layer support the error detection, healing and flow control. Network layer is utilized quality of service, congestion control and routing mechanism. Data link consists of MAC and LLC layers which provides link monitoring, frame size, multiple accesses and to minimize the collision with neighbor nodes. Physical layer provides modulation scheme, data rate control, bit error rate and bit coding scheme. Power management plan describes that how sensor node use power. For example node can go to sleep mode once it receive signal, this will help for power saving as well as stop duplication of data receiving. Mobility management plan note the mobility of sensor node and help in routing. Every node must keep track of its neighbor nodes.

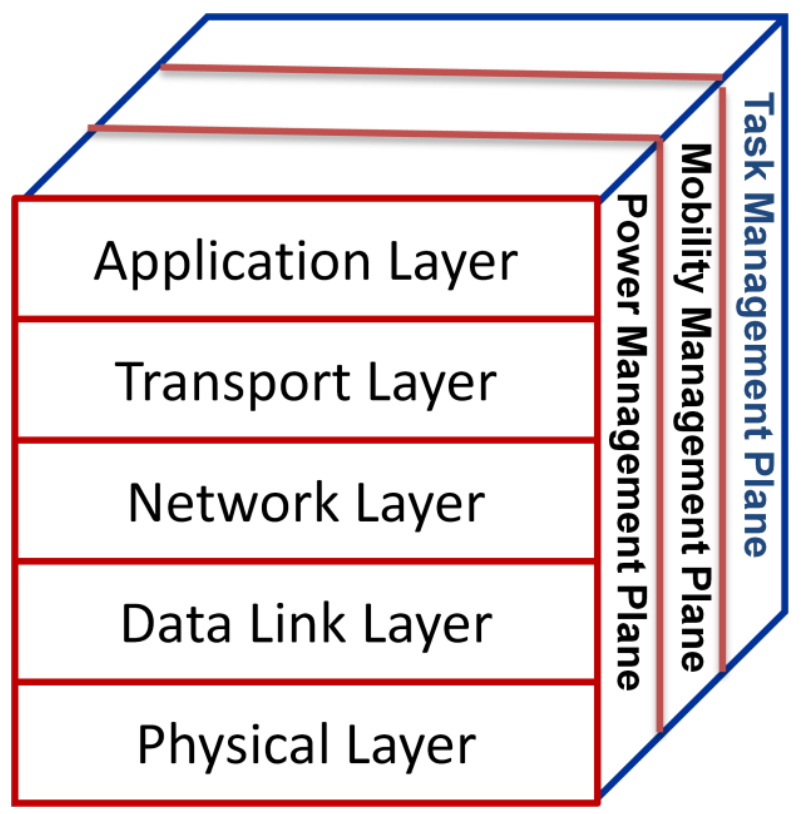

Fig 2: Wireless sensor network- Protocol stack.

Task management plan is used to elaborate the task to every node because every node is not participating equally in one time transmission.

\section{ROUTING CHALLENGES AND DESIGN ISSUES}

Sensor network protocol influenced by many factors, discussion of these issues is important because it leads us proper selection of protocol and design of algorithm. Routing in wireless sensor network is very challenging because of following design issues:

\subsection{Node Deployment}

Deployment of wireless sensor node could be done in two ways i.e. manual deployment and random deployment. In manual deployment, nodes are deployed on predefined position. They are deployed in shorter distance like 10's of feet from each other [3]. For example nodes deployed in factory, home etc. In such cases data is routed in predefined path. While in random deployment nodes are thrown in shape of bunch. For example nodes throwing from plane or ship in enemy field or under water. In this situation optimal clustering is necessary to allow connectivity and energy efficiency.

\subsection{Data Routing Method}

In WSN one of three methods could be used. In the continuous delivery model [4], every node sends data after some time sporadically. In event driven routing method data is transmitted on occurrence of an event. While in Query driven routing method, data is collected by sink by issuing the query when it needed. And in Hybrid technique two or more different techniques are used collectively.

\subsection{Node Heterogeneity}

In some situation nodes in WSN are homogeneous while in some case these are heterogeneous in terms of energy, band width and memory. Theses heterogeneous node in a same network behaves differently. These set of heterogeneous nodes creates different types of technical issues like different data rate, multiple data routing method etc. So these heterogeneous nodes create more challenges for routing strategy.

\subsection{Fault Tolerance}

Sensor nodes in WSN are consuming very less energy even then few node become failures because of battery dry up. Some nodes failed due to environmental damage. Fault tolerance is an ability of network that it sustains its performance for how much time and on how much numbers of sensing node failure.

\subsection{Data Aggregation}

Sensor node may generate redundant data. To minimize the communication load, WSN uses the data aggregation technique. Different techniques could be used for aggregation like suppression; $\min , \max$ and average function are performed. Data aggregation technique is used to save the energy, as more energy used in communication then computation. Sometime this goal will be achieved by signal processing (reducing the noise and more accurate signal). It is called data fusion

\subsection{Quality of Service}

The functionalities of WSN need the different Quality of Service (QoS) parameters for data transferring. QoS demand for a particular application of WSN normally different from any other application demands. So single protocol cannot satisfies the demands of all kind of applications. For illustration, a routing protocol might be intended to extend the network lifetime [5]. 
There are many other design issues that may challenging for routing protocol development, like Autonomy, Energy efficiency, Scalability, Resilience, Mobility adoptability.

\section{ROUTING PROTOCOLS}

On basis of different design factors and requirement for different application, routing protocol for sensor network can be subdivided in to four categories:

- Data centric

- Hierarchical

- $\quad$ Location based

- QoS based

\subsection{Data Centric Protocol}

In many applications of wireless sensor network, we cannot use the unique global ID (IP addresses) for sensor node. Therefore data is transmitted from one node to all other nodes in deployment region. This leads towards high energy consumption and congestion in WSN. Routing protocols is used to select a set of sensor nodes and utilize data aggregation during the relaying of data have been considered. This thought has led to data-centric routing, which is diverse from conventional address-based routing where routes are formed among addressable nodes handled in communication stack of network layer [4]. Too many protocols are developed on data centric approach. Some of them are as follows:

\subsubsection{Flooding and Gossiping}

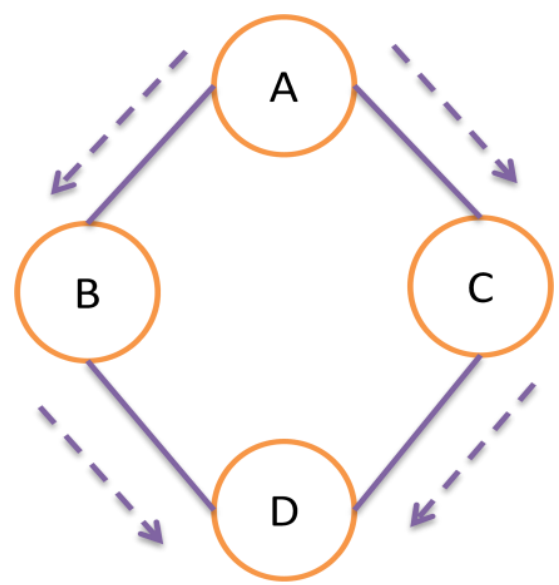

Fig 3: Data aggregation in flooding

Flooding and gossiping [7] are two classical mechanisms to relay data in sensor networks without the need for any routing algorithms and topology maintenance.

In flooding a node that receive data, send it to its all neighbor nodes until data reached at its destination. Gossiping is with little variation of flooding technique. Receiving node send the data to one randomly selected node. That node selects another node randomly for forwarding the data. Figure 3 from [7] shows the implosion problem. Where node A starts by flooding its data to all neighbors, node $\mathrm{D}$ received two identical copies of same data Gossiping resolve the issue of implosion by randomly selection of one forwarding node, but it causes the delay.

\subsubsection{Sensor Protocol for Information via Negotiation (SPIN)}

In SPIN protocol, receiving node send an advertise message to its entire neighbor. Only interested nodes reply it via REQ message. Then actual data is sent to those nodes. As shown in fig 4, three types of messages are used for one transaction, ADV advertisement message, REQ request message and actual DATA message. SPIN solves the classical problem of flooding like redundant information passing. It also achieves the lot of energy efficiency. However it does not guarantee the delivery of data. For example a node advertise a message if the interesting node is not in its neighbor, no REQ message received and data delivery to interesting node is not possible. SPIN is not a good choice for such system where reliable data delivery is required.

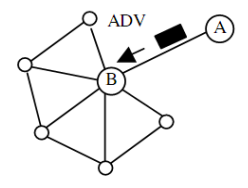

(a)

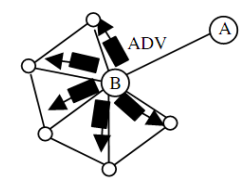

(d)

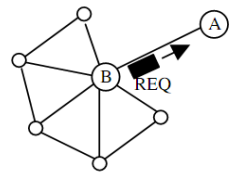

(b)

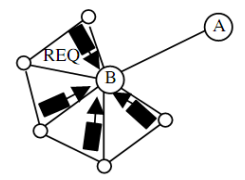

(e)

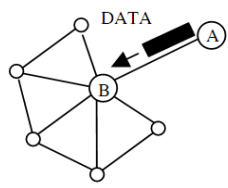

(c)

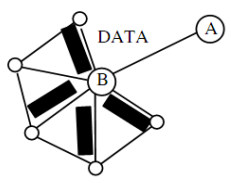

(f)
Fig 4: SPIN (Data Advertisement, Data Request and DATA flow) [7]

\subsubsection{ACQUIRE}

Active Query forwarding In sensor networks (ACQUIRE) is an on-demand query mechanism. Sink node send query when it need data. Each node tries to answer the query by using info and forward the query to another node. The querying mechanism works as follows: the query is forwarded by the sink and each node receiving the query, tries to respond partially by using its pre cached information and forward it to another sensor. If the pre-cached information is not latest, the nodes collect data from its neighbors within a look-ahead of d hops [9]. Once the query is resolved completely, it is sent back to sink node via reverse path or shortest path. If $d=$ network diameter then ACQUIRE works similar to flooding. If $\mathrm{d}$ is too small then query has to travel more. ACQUIR proves by mathematical modeling. A best value of parameter $\mathrm{d}$ is measured for a grid of sensors where every node has four nearby neighbors [9]. Direct Diffusion is another approach for query driven protocol, that support only the simple query but ACQUIRE can support complex queries as well. Complex query are in which responses can be provided by many nodes.

\subsection{Hierarchical Protocols}

As the network grows this is impossible for single tier network to provide the functionalities without delay. To cover the large area with wireless sensor network, clustering is introduced in some routing techniques. The main aim of hierarchical routing is to efficiently maintain the energy consumption of sensor nodes by involving them in multi-hop communication within a particular cluster and by performing data aggregation and fusion in order to decrease the number of transmitted messages to the sink [4]. Hierarchical routing protocols are as under: 


\subsubsection{Low Energy Adaptive Clustering}

\section{Hierarchical (LEACH) Routing Protocol}

It is one of the most popular hierarchical routing protocols for WSN. LEACH is cluster based protocol, in which any node can be chosen randomly as cluster head. LEACH contains randomized rotation of the high-energy cluster-head position such that it moves between the different sensors in order to not exhaust the battery of a single sensor [10]. In LEACH, cluster head compress data received from node and send aggregated data to sink node. LEACH is distributed routing protocol, and no need if global knowledge required for network. While in short comings, selection of cluster head is an issue. Synchronization of all nodes with their cluster head in every round is an issue for LEACH protocol.

\subsubsection{Power-efficient Gathering in Sensor} Information Systems (PEGASIS)

It is an improve version of LEACH Protocol. The main idea in PEGASIS is for every node have to receive and transmit to near neighbors and get turns being the head for transmission to the base station BS [11]. Rather than forming multiple clusters, PEGASIS forms chains from sensor nodes so that each node transmits and receives from a neighbor and only one node is selected from that chain to transmit to the base station (sink). As shown in figure 5, node $\mathrm{c} 2$ is leader of chain that send the data to base station. Once $\mathrm{C} 2$ receive data from $\mathrm{c} 0$ and $\mathrm{c} 1$, it pass signal to $\mathrm{c} 4$. After receiving data from $\mathrm{c} 4$ and $\mathrm{c} 3, \mathrm{c} 2$ aggregate it and send to base station.

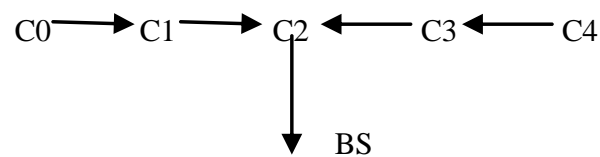

Fig 5:.PEGASIS chain

Performance of PEGASIS is much better than LEACH through the eliminating the overhead cause by the dynamic cluster formation in LEACH. However PEGASIS introduces excessive delay for distance node on chain. In addition single leader can cause a bottle neck problem.

\subsubsection{Threshold Sensitive Energy Efficient sensor} Network Protocol (TEEN)

Hard Threshold (HT): This is a threshold [12] value for the sensed attribute. It is the absolute value of the attribute beyond which, the node sensing this value must switch on its transmitter and report to its cluster head.

Soft Threshold (ST): This is a small change [12] in the value of the sensed attribute which triggers the node to switch on its transmitter and transmit.

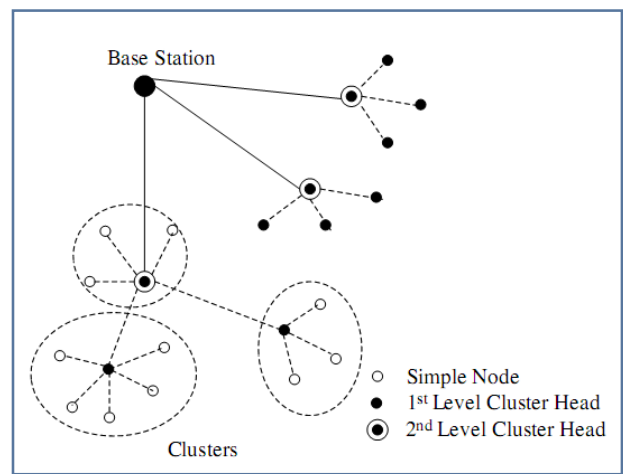

Fig 6: Cluster based hierarchical structure [12].
Node sense data continuously and wait until first time value reached up to hard threshold. Nodes save this value as stored variable (SV). It transmit this value to next node when the current value is greater than threshold or current value varies from SV equal to or greater from soft thresh hold.

To control the number of packet, hard and soft threshold levels can also be adjusted. TEEN is not good for applications where regular monitoring is required. Data transmission is done less frequently, so energy consumption is very less as compared to proactive approaches.

\subsection{Location Based Protocols}

Many energy aware routing protocols depends location of sensor node. They needed the distance between two nodes for energy consumption calculation. If the distances between nodes are known, query can be diffused only to the particular unit which will eliminate number of transmission significantly [4]. Such routing protocols are called location based routing protocols.

\subsubsection{Geographic adaptive fidelity $(G A F)$}

It is energy aware location based routing protocol. GAF mainly establish for MANET. Each node knows its position by GPS. It establish virtual grid for covered area. Each node uses its GPS-indicated location to associate itself with a point in the virtual grid. Nodes associated with the same point on the grid are considered equivalent in terms of the cost of packet routing [4]. As shown in fig 7. [13], node 1 can

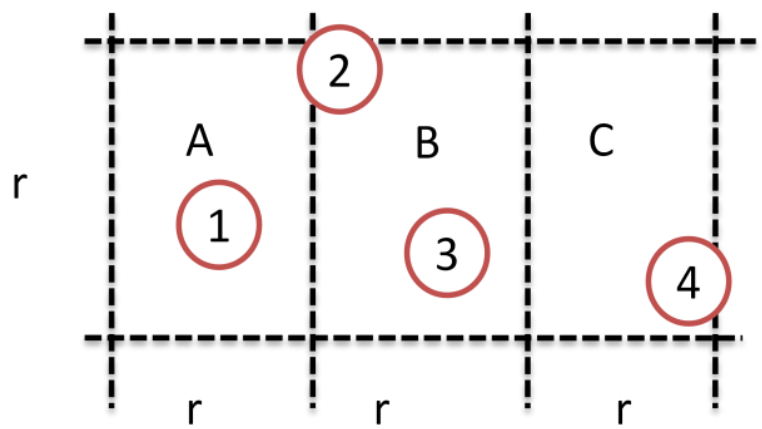

Fig 7: Virtual grid of GAF

transmit to any node 2, 3, 4 as node 2, 3, 4 are equivalent. Any of two nodes can sleep without affecting the routing fidelity.

GAF works on three states model, discovery, activate and sleep. First of sending node discover the all nodes of next virtual region. Then activate the path with one node and then in virtual region rest of node can sleep. Sleeping neighbor can adjust their sleeping time to keep the routing fidelity. In terms of latency, packet loss and increased life time, GAF results are as equal as of ad hoc routing protocol.

Other routing protocols in domain of location based routing protocols are MECN (minimum energy communication network) and GEAR (geographically and energy aware routing protocol).

\subsubsection{Geographic and Energy Aware Routing Protocol}

The geographic and energy-aware routing (GEAR) is another location based protocol. GEAR is area cost routing protocol. Instead of selection for single forward node as in GAF, it select the nearby all nodes toward destination. GEAR considers only a certain region of the network rather than 
flooding the entire network. The idea is to restrict the number of interests in Directed diffusion by only considering a certain region rather than sending the interests to the whole network. GEAR compliments Directed Diffusion in this way and thus conserves more energy. In GEAR, each node keeps a projected cost and a learning cost of getting the purpose through its neighbors [4]. The estimated cost is a combination of residual energy and distance to destination. The learned cost is a sophistication of the estimated cost that accounts for routing around holes in the network [4]. Learned cost is propagated one hop back every time a packet reaches the sink. A hole arises if node does not have near the target region. The estimated cost equal to the learned cost there are no holes. The learned cost is propagated one hop back every time a packet reaches the destination so that route setup for next packet will be adjusted.

\subsection{QoS-Aware Protocols}

Minimizing energy consumption is one consideration of routing protocol, while many other features of quality of service are delay, reliability, life time, fault tolerance.

\subsubsection{Sequential Assignment Routing (SAR)}

Sequential assignment routing (SAR) is routing protocol of sensor network, in which first time idea of QoS is used as routing decision. It uses the multipath and table driven approach is a table-driven approach to obtain targets of fault tolerance and energy efficiency. It establishes QoS metric by one step neighbor's information toward sink node. It considers this path and priority level of data packet. In this way multiple path from node to sink are established. SAR selects one of these paths on basis of QoS and energy available resources. Failure recovery is done by enforcing routing table consistency between upstream and downstream nodes on each path. Any local failure causes an automatic path restoration procedure locally. Simulation results show that SAR offers less power consumption than the minimumenergy metric algorithm, which focuses only the energy consumption of each packet without considering its priority. SAR maintains multiple paths from nodes to sink. SAR provide fault tolerance and easy recovery from failure but this is also have overhead of maintaining the table. When number of nodes increases, it is almost impossible to store the information of all nodes of all states.

\subsubsection{SPEED}

This is QoS routing protocol that provides soft real time end to end guarantee. Each node store information of neighbor and use it for finding the route. SPEED protocol ensures the speed of data from nodes to sink. SPEED strive [14] to ensure a certain speed for each packet in the network so that each application can estimate the end-to-end delay for the packets by dividing the distance to the sink by the speed of the packet before making the admission decision. Moreover, SPEED can provide congestion avoidance when the network is congested [14]. The routing module in SPEED is called stateless geographic non-deterministic forwarding (SNFG) and works with four other modules at the network layer [4]. Its algorithm is simple so total energy transmission is less, packet over head is less.

\section{CONCLUSION}

Applications of wireless sensor network are increasing because of smart, inexpensive, light weight sensor nodes. This paper presents what are the factors by which a routing protocol is selected. Energy consumption is major concern in all existing protocol for wireless sensor network. It effects the lifetime of network. In most of cases, when first mode died because of low energy, rest of nodes in running network died. In some applications nodes are deployed on such locations where we could not change their batteries or even could use the rechargeable batteries.

No single routing protocol available that can be used for all kind of wireless sensor network applications. Further research would be required to improve the lifetime and QoS parameter for wireless sensor network.

\section{ACKNOWLEDGMENTS}

The author would like to thank Dr. Nadeem Javed, Professor COMSATS, ComSense (Communication over Sensors) Research Group. Also special thanks to Mr. Mateen Yaqoob for his valuable suggestion and guideline in material searching and writing the paper.

\section{REFERENCES}

[1] C. Perkins, Ad Hoc Networks, Addison-Wesley, Reading, 2000.

[2] I.F. Akyildiz, W. Su, Y. Sankarasubramaniam, E. Cayirci, Wireless sensor networks: a survey, ELSEVIER, Computer Networks vol.38, 2002, 393-422.

[3] C. Intanagonwiwat, R. Govindan, D. Estrin, Directed diffusion: a scalable and robust communication paradigm for sensor networks, Proceedings of the ACM MobiCom'00, Boston, MA, 2000, 56-67.

[4] Akkaya, K.; Younis, M. A Surveybon Routing Protocols for Wireless Sensor networks. Ad Hoc Netw. 2005, vol. 3, 325-349.

[5] Luis Javier Garcia Villalba, Ana Lucila Sandoval Orozco, Alicia Triviño Cabrera and Cláudia Jacy Barenco Abbas, Review Routing Protocols in Wireless Sensor Networks, Sensors 2009, vol. 9, 8399-8421

[6] W. Heinzelman, A. Chandrakasan, H. Balakrishnan, Energy-efficient communication protocol for wireless sensor networks, in: Proceeding of the Hawaii International Conference System Sciences, Hawaii, January 2000.

[7] W.R. Heinzelman, J. Kulik, H. Balakrishnan, Adaptive protocols for information dissemination in wireless sensor networks, Proceedings of the ACM MobiCom'99, Seattle, Washington, 1999, 174-185.

[8] S. Hedetniemi, A. Liestman, A survey of gossiping and broadcasting in communication networks, Networks vol.18 (1988) 319-349.

[9] N. Sadagopan et al., The ACQUIRE mechanism for efficient querying in sensor networks, in: Proceedings of the First International Workshop on Sensor Network Protocol and Applications, Anchorage, AK, May 2003.

[10] W. Heinzelman, A. Chandrakasan, H. Balakrishnan, Energy-efficient communication protocol for wireless sensor networks, in: Proceeding of the Hawaii International Conference System Sciences, Hawaii, January 2000.

[11] S. Lindsey, C.S. Raghavendra, PEGASIS: power efficient gathering in sensor information systems, in Proceedings of the IEEE Aerospace Conference, Big Sky, Montana, March 2002.

[12] A. Manjeshwar, D.P. Agrawal, TEEN: a protocol for enhanced efficiency in wireless sensor networks, in: 
Proceedings of the 1st International Workshop on Parallel and Distributed Computing Issues in Wireless Networks and Mobile Computing, San Francisco, CA, April 2001.

[13] Y. Xu, J. Heidemann, D. Estrin, Geography-informed energy conservation for ad hoc routing, in: Proceedings of the 7th Annual ACM/IEEE International Conference on Mobile Computing and Networking (MobiCom01), Rome, Italy, July 2001.

[14] Shio Kumar Singh, M P Singh and D K Singh, Routing Protocols in Wireless Sensor Networks A Survey, International Journal of Computer Science \& Engineering Survey (IJCSES) Vol.1, No.2, November 2010 . 